\title{
Animated molecular dynamics simulations of hydrated caesium-smectite interlayers
}

\author{
Rebecca Sutton* and Garrison Sposito \\ Geochemistry Department, Earth Sciences Division, Mail Stop 90/1116, Lawrence Berkeley \\ National Laboratory, Berkeley, CA 94720-1116.E-mail: rsutton@nature.berkeley.edu
}

Received 22nd May 2002, Accepted 11th September 2002

Published on the Web 24th September 2002

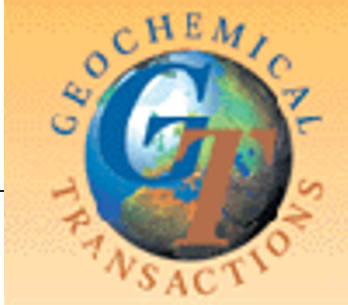

Computer animation of center of mass coordinates obtained from 800 ps molecular dynamics simulations of Cs-smectite hydrates (1/3 and 2/3 water monolayers) provided information concerning the structure and dynamics of the interlayer region that could not be obtained through traditional simulation analysis methods. $\mathrm{Cs}^{+}$formed inner sphere complexes with the mineral surface, and could be seen to jump from one attracting location near a layer charge site to the next, while water molecules were observed to migrate from the hydration shell of one ion to that of another. Neighboring ions maintained a partial hydration shell by sharing water molecules, such that a single water molecule hydrated two ions simultaneously for hundreds of picoseconds. Cs-montmorillonite hydrates featured the largest extent of this sharing interaction, because interlayer ions were able to inhabit positions near surface cavities as well as at their edges, close to oxygen triads. The greater positional freedom of $\mathrm{Cs}^{+}$within the montmorillonite interlayer, a result of structural hydroxyl orientation and low tetrahedral charge, promoted the optimization of distances between cations and water molecules required for water sharing. Preference of $\mathrm{Cs}^{+}$for locations near oxygen triads was observed within interlayer beidellite and hectorite. Water molecules also could be seen to interact directly with the mineral surface, entering its surface cavities to approach attracting charge sites and structural hydroxyls. With increasing water content, water molecules exhibited increased frequency and duration of both cavity habitation and water sharing interactions. Competition between $\mathrm{Cs}^{+}$and water molecules for surface sites was evident. These important cooperative and competitive features of interlayer molecular behavior were uniquely revealed by animation of an otherwise highly complex simulation output.

\section{Introduction}

The high bioavailability and long halflives of radioactive isotopes of Cs make them a significant environmental hazard. $^{1-3}$ To isolate this hazard, nuclear waste containment facilities may be separated from host bedrock by compacted bentonite liners, ${ }^{2}$ shown in laboratory experiments to slow the diffusion of $\mathrm{Cs}^{+1,2,4-7}$ The smectite minerals making up these liners are $2: 1$ layer type clay minerals. ${ }^{8}$ Each layer consists of an alumina (dioctahedral) or magnesia (trioctahedral) sheet sandwiched between two silica tetrahedral sheets. The arrangement of silica tetrahedra in sheets results in the creation of a network of six-oxygen rings, at the center of each a distorted hexagonal cavity, across the surface of the mineral. Isomorphic substitution of $\mathrm{Mg}$ for $\mathrm{Al}$, or $\mathrm{Li}$ for $\mathrm{Mg}$, results in negative octahedral charge sites, while substitution of $\mathrm{Al}$ for $\mathrm{Si}$ creates negative tetrahedral charge sites. These charge sites are balanced by cations in the interlayer which attract waters of hydration to this region, causing the clay mineral to swell. Although Cssmectite hydrates have been studied for many years, ${ }^{9}$ little detailed information about the mechanisms involved in $\mathrm{Cs}^{+}$ adsorption, hydration, and diffusion has been adduced from experimental findings. Molecular simulation thus has proven to be a useful adjunct to experimental work concerning the smectite interlayer. ${ }^{10-15}$

A number of XRD studies show that the layer spacing of Cs-smectites remains in the range 11.9-12.5 $\AA$ after exposure to water vapor at any relative humidity, ${ }^{9,16-20}$ or even after immersion in an aqueous solution. ${ }^{9}$ Water vapor adsorption isotherms ${ }^{16-20}$ indicate increasing hydration of Cs-smectite with increasing relative humidity, but Calvet ${ }^{17}$ and Prost ${ }^{18}$ argued that most of the water adsorbed resides in micropores, not in the interlayer region. Indeed, measurements of the micropore volume in homoionic montmorillonites ${ }^{21}$ have established the extensive microporosity of Cs-smectites. Calvet ${ }^{17}$ and Prost $^{22}$ suggested that the $12.4 \AA$ layer spacing in Cs-smectite was achieved and stabilized after the adsorption of only about $1.2-1.4 \mathrm{H}_{2} \mathrm{O}$ per unit cell of the clay mineral, well below the nominal monolayer water content of $4 \mathrm{H}_{2} \mathrm{O}$ per unit cell observed for smectites containing the strongly hydrating $\mathrm{Li}^{+}$cation. ${ }^{17,19}$ Our recent Monte Carlo (MC) simulations of model Cs-smectite hydrates at low water contents ${ }^{15}$ confirmed this speculation, showing that the $12.4 \AA$ hydrate of Cs-smectite likely contains no more than $2.7 \mathrm{H}_{2} \mathrm{O}$ per unit cell (about 2/3 monolayer).

The Cs-smectites we investigated were (Table 1): Cshectorite with 1.3 and $2.7 \mathrm{H}_{2} \mathrm{O}$ per unit cell $(1 / 3$ and $2 / 3$ water monolayer), Cs-beidellite with $1 / 3$ water monolayer, and Cs-montmorillonite with $1 / 3$ and $2 / 3$ water monolayer. Caesium-smectites with 1.0, 1.31, 0.844, and 0.75 monolayers of water also were modeled for up to 10 million $\mathrm{MC}$ steps, but did not meet our MC equilibration criteria of achieving both a minimum average potential energy and a stable layer spacing. That simulations containing more water molecules were inherently unstable is not surprising given experimental data indicating Cs-smectites have low interlayer water contents. ${ }^{17,22}$

The MC simulations were followed by molecular dynamics (MD) simulations. ${ }^{15}$ These latter results were analyzed for average properties, such as interlayer water self-diffusion coefficients, as well as for individual molecular properties, such as plots of $x y z$ coordinates sampled by specific $\mathrm{Cs}^{+}$ ions or by water molecules. These traditional simulation outputs were not able to characterize the full complexity of interactions among cations, water molecules, and mineral 
Table 1 Layer spacing and molar potential energy of water (MC simulation) ${ }^{15}$ and self-diffusion coefficients for interlayer water (MD simulation) in Cs-smectite hydrates

\begin{tabular}{|c|c|c|c|c|}
\hline Hydrate & MC steps ${ }^{a}$ & Layer spacing/Å & $\begin{array}{l}\text { Potential energy of water/ } \\
\mathrm{kJ} \mathrm{mol}^{-1} b\end{array}$ & $\begin{array}{l}\text { Self-diffusion coefficient, } \\
D_{\mathrm{w}} / 10^{-9} \mathrm{~m}^{2} \mathrm{~s}^{-1 c}\end{array}$ \\
\hline \multicolumn{5}{|l|}{$1 / 3$ water monolayer } \\
\hline Cs-hectorite & $10^{6}$ & $12.37 \pm 0.08$ & $-47.43 \pm 3.53$ & $0.229 \pm 0.001$ \\
\hline Cs-beidellite $^{d}$ & $4 \times 10^{6}$ & $12.31 \pm 0.10$ & $-42.98 \pm 4.58$ & $0.342 \pm 0.002$ \\
\hline Cs-montmorillonite & $10^{6}$ & $12.46 \pm 0.09$ & $-32.33 \pm 4.04$ & Nonlinear ${ }^{\mathrm{e}}$ \\
\hline \multicolumn{5}{|l|}{ 2/3 water monolayer } \\
\hline Cs-hectorite & $3.0 \times 10^{6}$ & $12.41 \pm 0.07$ & $-46.56 \pm 2.00$ & $0.685 \pm 0.006$ \\
\hline Cs-montmorillonite & $3.5 \times 10^{6}$ & $12.68 \pm 0.10$ & $-36.66 \pm 2.12$ & $1.168 \pm 0.006$ \\
\hline \multicolumn{3}{|l|}{ Bulk liquid water $f$} & $-35.4 \pm 0.2^{31}$ & $2.3^{32}$ \\
\hline \multicolumn{5}{|c|}{$\begin{array}{l}{ }^{a} \text { Monte Carlo steps required for convergence. }{ }^{b} \text { (Total potential energy of hydrate }- \text { total potential energy of clay mineral) } \div \text { moles of water } \\
\text { per simulation cell. }{ }^{13}{ }^{c} \text { Value calculated from linear regression of the mean-square displacement }(\mathrm{MSD}) \text { on elapsed time with } P=0.05 \text { confi- } \\
\text { dence interval. }{ }^{15}{ }^{d} \text { This hydrate exhibited MC convergence only at the lower water content. }{ }^{15} \text { This hydrate did not produce a linear relationship } \\
\text { between MSD and elapsed time. }{ }^{15}{ }^{f} \mathrm{MCY} \text { water }{ }^{33} \text { yields } D_{\mathrm{w}}=2 \times 10^{-9} \mathrm{~m}^{2} \mathrm{~s}^{-1} \text {. }\end{array}$} \\
\hline
\end{tabular}

surface sites. To continue analysis of the MD results, we constructed animations of molecular motions in the five Cssmectite hydrate systems to examine the effects of charge site and hydration state on the behavior of interlayer species. Stereo-viewing and animated motion have been shown to increase comprehension of three dimensional structures. ${ }^{23}$ Because humans understand and navigate through a dynamic, three dimensional world, depiction of MD animations as a collection of spheres in three dimensional space that change position over time is a familiar metaphor that is intuitive to most viewers. ${ }^{23}$ Other more traditional forms of presentation, such as a display of the entire path of a molecule over time, can result in a "tangled mess" that is not easy to interpret. ${ }^{23}$ Animations thus can be helpful in understanding the complex three dimensional relationships between interacting molecules over time.

\section{Methods}

\section{Model Cs-smectite hydrates}

The simulation cell used consists of two opposing halves of a rigid $2: 1$ clay layer surrounding an interlayer region

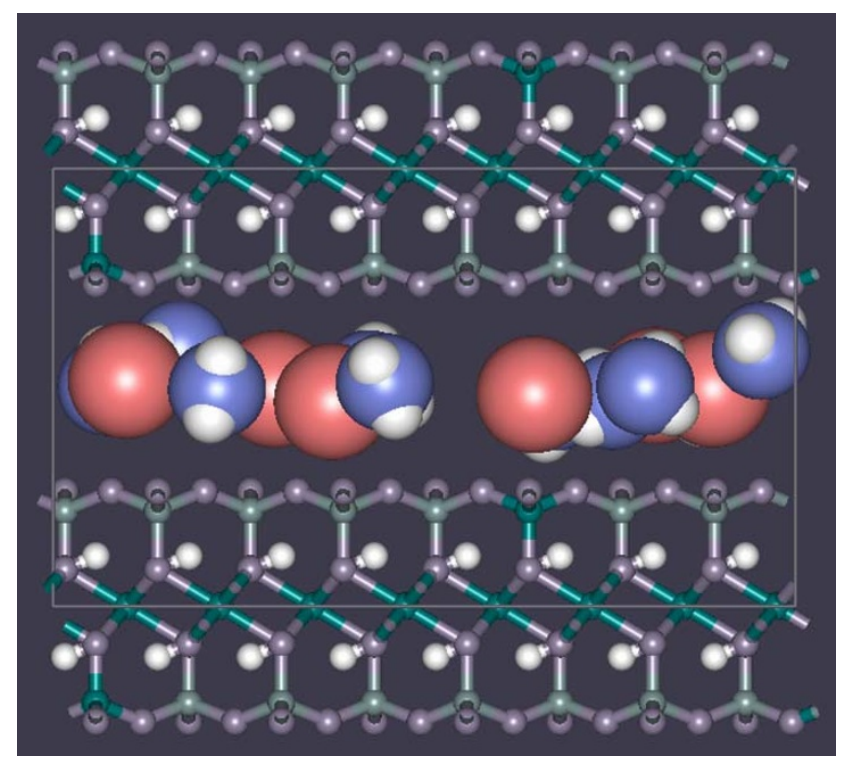

Fig. 1 A view of the simulation cell, outlined in grey, for Csmontmorillonite, $1 / 3$ water monolayer. Interlayer $\mathrm{Cs}^{+}$are represented by orange spheres, while interlayer water oxygens are represented by blue spheres and water hydrogens by white spheres. The surrounding mineral layers are displayed in ball and stick style, with the greyish purple balls representing the mineral oxygen atoms, white balls representing $\mathrm{H}$, grey balls representing $\mathrm{Si}$, and green balls representing Al. filled with rigid water molecules and $\mathrm{Cs}^{+}$ions (Fig. 1). The crystallographic unit cell formulae of the three Cs-smectites we modeled are:

Hectorite: $\mathrm{Cs}_{0.75}\left[\mathrm{Si}_{8}\right]\left(\mathrm{Mg}_{5.25} \mathrm{Li}_{0.75}\right) \mathrm{O}_{20}(\mathrm{OH})_{4}$ Beidellite: $\mathrm{Cs}_{0.75}\left[\mathrm{Si}_{7.25} \mathrm{Al}_{0.75}\right]\left(\mathrm{Al}_{4}\right) \mathrm{O}_{20}(\mathrm{OH})_{4}$

Montmorillonite: $\mathrm{Cs}_{0.75}\left[\mathrm{Si}_{7.75} \mathrm{Al}_{0.25}\right]\left(\mathrm{Al}_{3.5} \mathrm{Mg}_{0.5}\right) \mathrm{O}_{20}(\mathrm{OH})_{4}$

Hectorite is a trioctahedral 2:1 clay mineral, whereas montmorillonite and beidellite are dioctahedral. ${ }^{12}$ Moreover, hectorite shows charge substitution exclusively in the octahedral sheet, whereas beidellite contains only tetrahedral charge substitution. Montmorillonite has both types of charge substitution, with twice as much octahedral as tetrahedral layer charge. Our simulation cell contains eight unit cells, and has lateral $(a b)$ dimensions $21.12 \times 18.28 \AA^{2}$. The layer spacing is determined for each simulation system individually during MC equilibration.

Interactions between the interlayer species, and between them and atoms in the clay layers, are represented parametrically by a MCY-type potential function: ${ }^{11}$

$$
U=\sum_{i=1}^{N} \sum_{j>i}^{N}\left[\frac{q_{i} q_{j}}{r_{i j}}-A_{i j} \mathrm{e}^{-B_{i j} r_{i j}}+C_{i j} \mathrm{e}^{-D_{i j} r_{i j}}\right]
$$

where $U$ is the potential energy of the system, $i$ and $j$ are sites on atoms within the simulation cell, $r_{i j}$ is the distance between sites $i$ and $j, q$ represents atom charge, and $A, B, C$, and $D$ are van der Waals interaction parameters. We used this form of potential function because it has led to accurate modeling of experimentally accessible quantities such as layer spacing and interlayer species configuration and mobility. ${ }^{10-15}$ In addition, the MCY potential function for the water-water interaction is optimized on dimer structures ${ }^{25}$ and, therefore, does not enforce the tetrahedral configuration of bulk water as strongly as do other, more empirically-based water-water potential functions. This fact may explain its enhanced ability to model water molecules in the especially constrained geometry of the clay interlayer region. ${ }^{10,11,13}$ Van der Waals parameters for the Cs-O interaction were obtained through conversion of Lennard-Jones (LJ) (6-12) potential function parameters taken from Smith and Dang, ${ }^{26}$ described previously. ${ }^{15}$ The MCY Cs-O interaction parameters were: $A=$ $51.0 \mathrm{~kJ} \mathrm{~mol}^{-1}, B=1.0850 \AA^{-1}, C=3.72 \times 10^{6} \mathrm{~kJ} \mathrm{~mol}^{-1}$, $D=4.2758 \AA^{-1}$. Van der Waals interactions for Cs-H, $\mathrm{Cs}-\mathrm{Al}$, and $\mathrm{Cs}-\mathrm{Si}$ atom pairs were assumed to be negligible.

\section{Monte Carlo and molecular dynamics simulations}

Allen and Tildesley $^{24}$ have outlined the general principles of MC and MD simulations of liquids. Chang et al. ${ }^{10,11}$ and Greathouse and Sposito ${ }^{12}$ have developed simulation methodologies customized for exploration of the interlayer 
region of 2:1 clay mineral hydrates. Detailed description of MC calculations performed to establish the equilibrated Cs-smectite hydrate structures has been provided previously. ${ }^{15}$ The program MONTE ${ }^{27}$ was used to run MC (N $\sigma \mathrm{T}$ ensemble) simulations on a variety of Cs-smectite hydrates at $300 \mathrm{~K}$ and $100 \mathrm{kPa}$ normal stress $(\sigma)$. Monte Carlo calculations began with a simulation cell that had a $14 \AA$ layer spacing and contained $6 \mathrm{Cs}^{+}$in the midplane of the interlayer along with several randomly placed water molecules. Three-dimensional periodic boundary conditions were applied. ${ }^{24}$ Simulation started with an optimization developed by Chang et al. ${ }^{10,11}$ consisting of 20,000 MC steps in which only water molecules were allowed to move, followed by another 20,000 steps in which water molecules could move and interlayer spacing could vary. After this optimization, all molecules were allowed to move in any direction, provided that the clay layer only moved about once for every five interlayer molecule movements. Information about total potential energy and layer spacing was collected every 500 steps. Systems were considered MC-equilibrated when convergence profiles of total potential energy and layer spacing provided minimum average values. The standard deviation of the average layer spacing was further required to be consistent with the precision of available experimental data on layer spacing. ${ }^{15}$

The program MOLDY ${ }^{28}$ was used to perform MD (NVE ensemble) simulations of the Cs-smectite hydrates which had converged under MC simulation. Monte Carlo-equilibrated configurations in which the layer spacing was held constant were used as initial coordinates for the MD calculations. With the rigid clay layers fixed in position, initial velocities were assigned randomly to interlayer molecules following a Maxwell-Boltzmann distribution. In order to stabilize the temperature at $300 \mathrm{~K}$, the simulations began with $20 \mathrm{ps}$ of temperature scaling ${ }^{28}$ using a 0.5 fs time step. Temperature scaling was performed for each molecular species individually, with rotational and translational kinetic energies scaled separately. After initial scaling, the MD simulations proceeded for $800 \mathrm{ps}$.

Molecular center-of-mass coordinates collected at $0.1 \mathrm{ps}$ intervals during the simulations were used as the input data for our animations. We used a custom animation tool to create a series of JPEG images, one for each set of coordinates, based on the center-of-mass information. The custom animation tool, created by the Lawrence Berkeley National Laboratory/National Energy Research Scientific Computing Center (LBNL/NERSC) Visualization Group ${ }^{29}$ in collaboration with the authors, runs on Windows or Unix/Linux platforms, and is based on OpenRM Scene Graph, an Open Source, cross-platform scene graph library. ${ }^{30}$ These images were then converted to MPEG-1 format using the MediaConvert tool and executed on a visualization server located in the Visualization Laboratory at LBNL.

\section{Results and discussion}

Molecular dynamics simulation and subsequent animation revealed complex and fascinating interactions between $\mathrm{Cs}^{+}$ ions, water molecules, and the charged clay surface. Short animations of twenty to fifty picoseconds $(\sim 1-2 \mathrm{MB})$ illustrating features relevant to the discussion below may be obtained from the links provided.

Animations of the five systems share some basic similarities of molecular behavior. For example, $\mathrm{Cs}^{+}$tend not to roam, but instead to hover near the charge sites recessed within the clay layer. When the ions do move, they display jump diffusional behavior, in which the ions vibrate in one place for some time, then jump to new locations (see the animation in Plate 1 , Cs-hectorite with $1 / 3$ water monolayer).

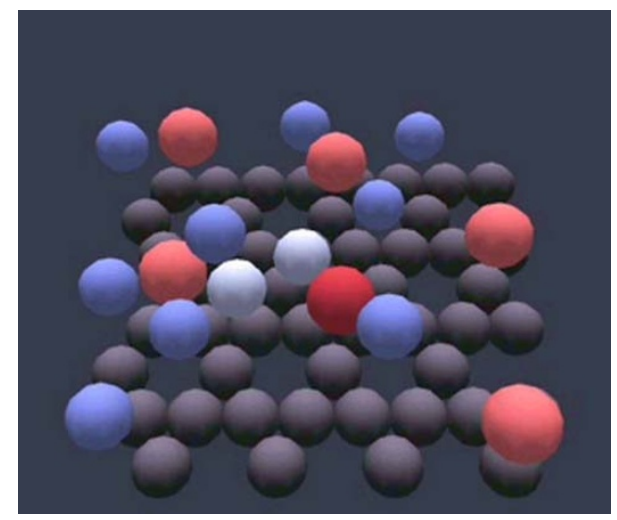

Plate 1 A 35 ps portion of the Cs-hectorite, 1/3 water monolayer MD simulation animation (85-120 ps of the 800 ps simulation). Interlayer $\mathrm{Cs}^{+}$are represented by orange spheres, while interlayer water molecules are represented by blue spheres. The greyish purple spheres symbolize the surface oxygen atoms of the lower clay layer surrounding this interlayer region. The rest of the atoms making up this lower clay layer, as well as all the atoms making up the upper clay layer, have been removed to improve the visibility of the interlayer region. Due to the periodic nature of the simulations, interlayer species which exit from one side of the simulation cell will reappear on the opposite side. The highlighted $\mathrm{Cs}^{+}$(red) can be seen to display jump diffusion, while the highlighted water molecule (lighter blue) in the center of the simulation cell is characterized by continuous diffusional behavior as it moves from one $\mathrm{Cs}^{+}$partial hydration shell to another. Another highlighted water molecule to the left can be seen visiting a surface cavity site for a short time. Click image or here to access the animation.

These motions tend to be confined to different locations around the charge sites attracting the cations. In contrast, the water molecules display continuous diffusion on the timescale of these animations. Both ions and water molecules move more frequently in the $2 / 3$ monolayer systems, a trend indicated for water molecules by an increased self-diffusion coefficient (Table 1). However, both $\mathrm{Cs}^{+}$and water molecule movements in the interlayer are substantially slower than in bulk ionic solution, where these two species have selfdiffusion coefficients of $2 \times 10^{-9} \mathrm{~m}^{2} \mathrm{~s}^{-1} \cdot 32,34$

Animations allow us to see changes in the structure of hydrating water molecules around interlayer $\mathrm{Cs}^{+}$over time. Water molecules move from one $\mathrm{Cs}^{+}$to another, but are rarely far from an interlayer ion (Plate 1). This behavior leaves small regions of the smectite surface empty in the $1 / 3$ monolayer simulations. Despite the weak coulomb field of $\mathrm{Cs}^{+}$and its large size, these ions are able to organize water molecules into partial hydration shells (water molecules within a radial distance of $<5 \AA$ from the cation, as shown by our MC radial distribution function calculations ${ }^{15}$ ). The number of water molecules within each shell varies substantially with time for each cation. The exchange behavior seen among $\mathrm{Cs}^{+}$waters of hydration contrasts with the results of MD calculations of similarly low water content Li-smectites, ${ }^{12}$ in which waters of hydration remained near the same ion for as long as 200 ps. The difference may be attributed to the smaller radius of the $\mathrm{Li}^{+}$ions, which results in stronger electrostatic attraction for water molecules, as well as to the shorter timespan of the Li-smectite simulations, ${ }^{12}$ which may not have been long enough to capture water exchange processes.

Bostick et $a l .{ }^{35}$ recently have interpreted $\mathrm{L}_{\mathrm{III}}$-edge EXAFS spectra of Cs-montmorillonite to suggest the presence of two types of $\mathrm{Cs}^{+}$-surface complex, distinguished by average Cs-O distances of 3.2 and $4.25 \AA$, respectively. They consider the complex with the shorter $\mathrm{Cs}-\mathrm{O}$ distance to be an outer sphere complex, fully hydrated and therefore separated from mineral surfaces by water molecules, while the complex with the longer Cs-O distance is thought to be an inner sphere complex, partially dehydrated and directly 
associated with a mineral surface. Their analysis suggests that in a fully-saturated Cs-montmorillonite, the majority of cations exist as outer sphere complexes directly coordinated by $2-3$ water molecules. Our simulations do not support a model of the interlayer which includes outer sphere $\mathrm{Cs}^{+}$ complexes. ${ }^{15}$ The cations modeled here have formed inner sphere complexes with the clay mineral surface and are surrounded by at least six oxygen atoms, yet have average $\mathrm{Cs}-\mathrm{O}$ distances of around $3 \AA,^{15}$ similar to the distance Bostick et $a l .{ }^{35}$ assign to an outer sphere complex. The large size of $\mathrm{Cs}^{+}$is inconsistent with the large energy of hydration needed to maintain an outer sphere complex near a charged mineral surface, as in the case of Li-montmorillonite. ${ }^{12}$

A highly variable Cs coordination environment caused by sample preparation may account for the discrepancies between our results and EXAFS interpretations. While simulations can focus specifically on the interlayer environment, spectroscopic analysis of Cs-smectites inevitably averages signals from $\mathrm{Cs}^{+}$in the interlayer, adsorbed to edge sites, and present in micropores. ${ }^{21}$ Careful sample preparation after $\mathrm{Cs}^{+}$saturation, involving a drying step designed to encapsulate the ion between surface cavities in the interlayer, followed by a $\mathrm{LiCl}$ rinsing step to remove less tightly bound ions, ${ }^{36,37}$ would result in a sample containing primarily inner sphere $\mathrm{Cs}^{+}$complexes residing in the interlayer of the smectite. A sample produced with this method would have a far less variable $\mathrm{Cs}^{+}$coordination environment, and could be useful as an end member describing inner sphere coordination for linear combination fitting of EXAFS spectra. ${ }^{35}$ Reinterpretation of the Bostick et al. ${ }^{35}$ data using this approach may result in a view of interlayer structure similar to that provided by our simulation.

The animations bring to light the phenomenon of water molecule sharing between two $\mathrm{Cs}^{+}$ions, an effect seen in all five simulations to varying degrees (Fig. 2). A shared water molecule may be defined as one which lies within the $<5 \AA$ hydration shells of two $\mathrm{Cs}^{+}$simultaneously for a period of hundreds of picoseconds or more. Shared water molecules remain in a fairly fixed position relative to the two $\mathrm{Cs}^{+}$they hydrate during this period of sharing. Our animations appear to be the first evidence for such an interaction, which likely requires the conditions of confined geometry, presence

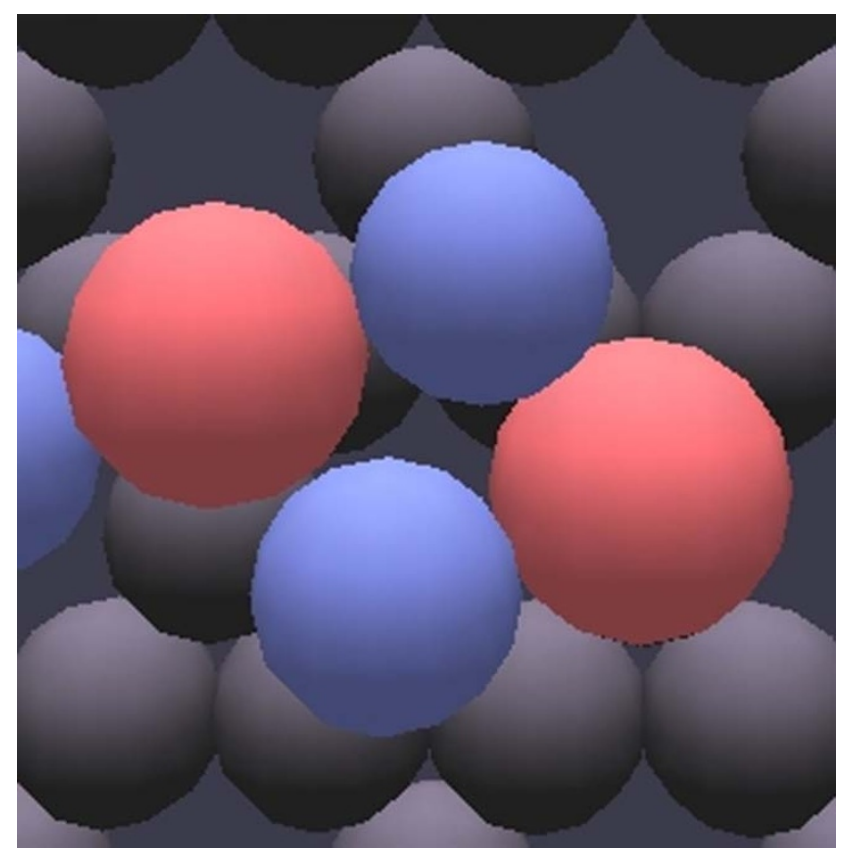

Fig. $2 \mathrm{~A}$ view in the $x y$ plane of the two $\mathrm{Cs}^{+}$-two water complex seen in the Cs-montmorillonite, 1/3 water monolayer animation (Plate 2).

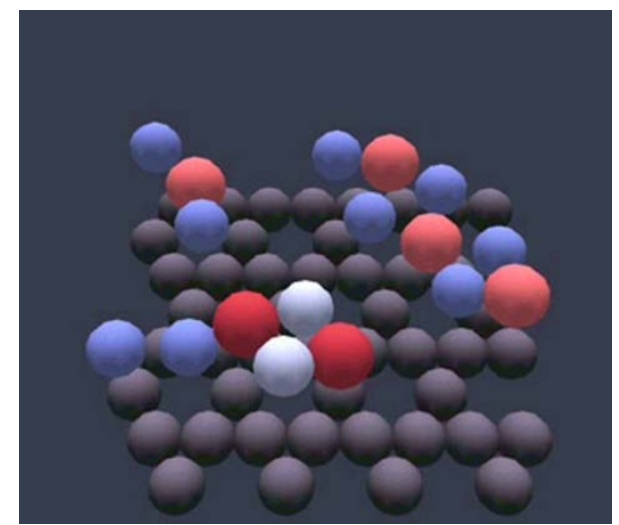

Plate 2 A 20 ps portion of the Cs-montmorillonite, 1/3 water monolayer MD simulation animation (65-85 ps). Interlayer species are represented as described in Plate 1 . The two highlighted $\mathrm{Cs}^{+}$(red) share the two highlighted water molecules (lighter blue). The molecules making up this two $\mathrm{Cs}^{+}-$two water complex are so strongly bound that they are observed to rotate as a unit during this animation. Interlayer water molecules do not enter six-oxygen cavities in this portion of the simulation. Click image or here to access the animation.

of surface charge, and close proximity of ions that are found in clay interlayers.

As was suggested for the EXAFS study mentioned above, ${ }^{35}$ a reinterpretation of existing NMR data on Cs-smectites ${ }^{38-41}$ also may be in order. These data, which informed the EXAFS analysis, ${ }^{35}$ also suggest that outer sphere $\mathrm{Cs}^{+}$ complexes exist in the interlayer region. ${ }^{38-41}$ This conclusion, not supported by molecular modeling, ${ }^{15}$ does not consider the possibility of two $\mathrm{Cs}^{+}$bound by one or more shared water molecules.

Cs-hectorite with $1 / 3$ water monolayer (Plate 1) exhibits the least water sharing, with two $\mathrm{Cs}^{+}$pairs (the top left and bottom right $\mathrm{Cs}^{+}$are one pair, and the middle two $\mathrm{Cs}^{+}$on either side of the simulation cell are another), each sharing one water molecule for hundreds of picoseconds. In contrast, Cs-montmorillonite with $1 / 3$ water monolayer (Plate 2) contains two pairs of $\mathrm{Cs}^{+}$that share two water molecules per pair over the entire simulation (two $\mathrm{Cs}^{+}$at the bottom of the simulation cell form one pair, while the other is made up of the middle two $\mathrm{Cs}^{+}$on either side of the simulation cell). The molecules making up the two $\mathrm{Cs}^{+}$-two water complex near the bottom of the Csmontmorillonite, $1 / 3$ water monolayer animation are so strongly bound that they can be seen to rotate as a unit (Plate 2). In addition, other $\mathrm{Cs}^{+}$pairs in this system share one or two water molecules, but only for a few hundred picoseconds or less. Such entrapment of the water molecules could underlie the nonlinear relationship between their mean-square displacement (MSD) and elapsed time noted for this system, ${ }^{15}$ which signaled the absence of selfdiffusion in the interlayer water (Table 1). Cs-beidellite with $1 / 3$ water monolayer (Plate 3 ) features three pairs of cations which participate in water sharing of one or two water molecules for substantial portions of the 800 ps simulation (cations to the left and right of the animation are one pair, cations to the top and bottom of the animation are another, and the third consists of the top cation and its neighbor to the lower left). Cs-beidellite with $1 / 3$ water monolayer may be considered to have an intermediate level of water sharing, as compared to the hectorite and montmorillonite systems, as it features more water sharing cation pairs than hectorite, but reduced number of shared water molecules and duration of water sharing per pair as compared to montmorillonite.

A difference in cation location on the clay surface caused by both the orientation of structural hydroxyl groups and the relative proportion of tetrahedral charge sites, may 


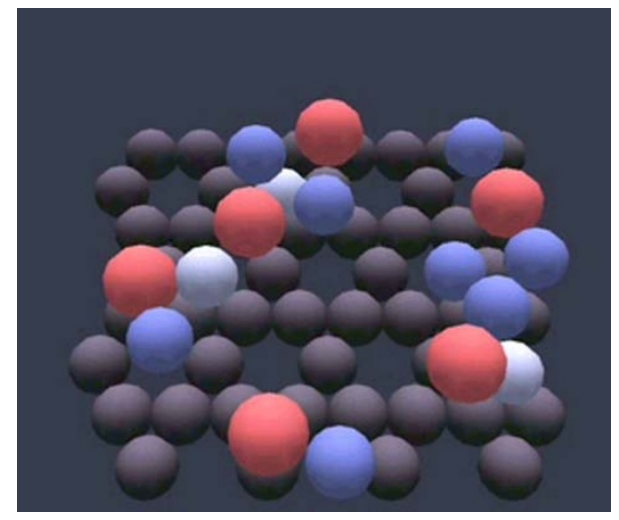

Plate 3 A 30 ps portion of the Cs-beidellite, 1/3 water monolayer MD simulation animation (350-380 ps). Interlayer species are represented as described in Plate 1. The three highlighted water molecules (lighter blue) reside within six-oxygen cavities of the lower clay surface for extended time periods. One of these water molecules, located near the top of the small view at the top left of the animation, and nearly hidden by a $2 \mathrm{Cs}^{+}-2$ water molecule complex, stays within the six-oxygen cavity for the $30 \mathrm{ps}$ length of this animation. Click image or here to access the animation.

account for the degree of water sharing present in each clay system. Our previous research ${ }^{15}$ noted a larger range of movement among the interlayer $\mathrm{Cs}^{+}$on montmorillonite, which we hypothesized to be a result of the disorder induced by the presence of both octahedral and tetrahedral charge (Fig. 3). Closer inspection of the Cs-montmorillonite, $1 / 3$ water monolayer system (Plate 2), relative to the hectorite and beidellite systems (Plates 1 and 3) reveals the specific cation motions which result in this greater variation in location. The cations within montmorillonite frequently hover over and dip into the cavities of the mineral surface, while the cations within hectorite and beidellite interlayers rarely stray from positions directly over oxygen triads found at the edges of these cavities. Entrapment of $\mathrm{Cs}^{+}$between opposing surface cavities is a natural process that can be induced in the laboratory through alternate wetting and drying procedures, and is the basis for standard methods used to determine structural surface charge. ${ }^{36,37}$ Our MC calculations, which provide the coordinates of the mineral layer used in the MD simulations and established a water content of less than a monolayer, are not constrained to achieve the layer registration necessary to encapsulate a cation between opposing surface cavities, nor can they be used to simulate the process of wetting and drying. As a result, our simulations do not lead to cation fixation within surface cavities.

The difference in $\mathrm{Cs}^{+}$behavior within the interlayer of the three minerals, which has an electrostatic explanation, may be contrasted with the behavior of the much smaller $\mathrm{Li}^{+}$ ion in similar low water content systems. ${ }^{12}$ The structural hydroxyl groups located at the bottom of the six-oxygen cavities of hectorite are oriented perpendicularly to the clay surface, such that the positive portion of this polar group repels the interlayer cation and discourages it from entering the cavity (Fig. 4). It is likely that this electrostatic repulsion is exaggerated in our simulations as a result of imposing a rigid clay structure, which prevents structural hydroxyl groups from relaxing into non-perpendicular orientations. The octahedral charge sites of hectorite, submerged within the clay layer, are too far away to counteract this repulsion effectively. $\mathrm{Cs}^{+}$thus maintain midplane positions in the interlayer as a result of the distant octahedral charge and structural hydroxyl repulsion (Fig. 4), as did $\mathrm{Li}^{+} .{ }^{12}$ The smaller size of $\mathrm{Li}^{+}$provides it with sufficient energy of hydration to form an outer sphere complex within hectorite, ${ }^{12}$ while the larger $\mathrm{Cs}^{+}$forms an inner sphere complex with the clay surface.

Though the structural hydroxyls of beidellite are oriented at a shallow angle relative to the surface, ${ }^{42}$ which reduces

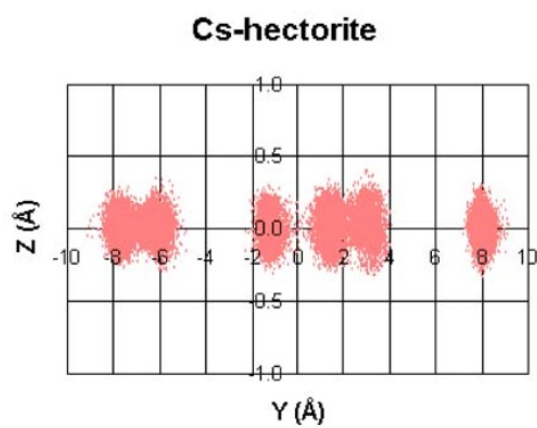

Cs-beidellite

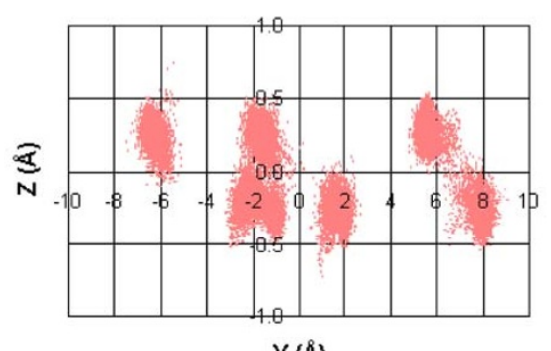

$Y(\AA)$

Cs-montmorillonite

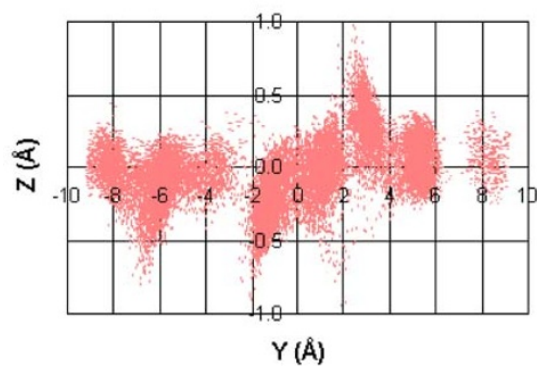

Fig. 3 Cumulative $z y$ coordinates of $\mathrm{Cs}^{+}$(first $400 \mathrm{ps}$ ) for Cs-smectites with $1 / 3$ water monolayer. The surrounding clay layers are located out of the figure at $\pm 1.52, \pm 1.44$, and $\pm 1.54 \AA$ along the $z$ axis for Cshectorite, Cs-beidellite, and Cs-montmorillonite, respectively.

electrostatic repulsion, the tetrahedral charge sites present in the mineral are located directly beneath some of the oxygen triads at the surface, making them attractive locations for interlayer cations (Fig. 4). Molecular modeling of Li-beidellite resulted in $\mathrm{Li}^{+}$positioned directly over oxygen triads. ${ }^{12}$ In contrast, cations within Cs-montmorillonite interlayers can inhabit surface cavities as well as positions near oxygen triads. Only the simulations of montmorillonite contain both structural hydroxyl groups with shallow orientations relative to the surface, ${ }^{42}$ and a small proportion of tetrahedral charge, conditions which allow $\mathrm{Cs}^{+}$to occupy positions within or near six-oxygen cavities and close to oxygen triads (Fig. 4).

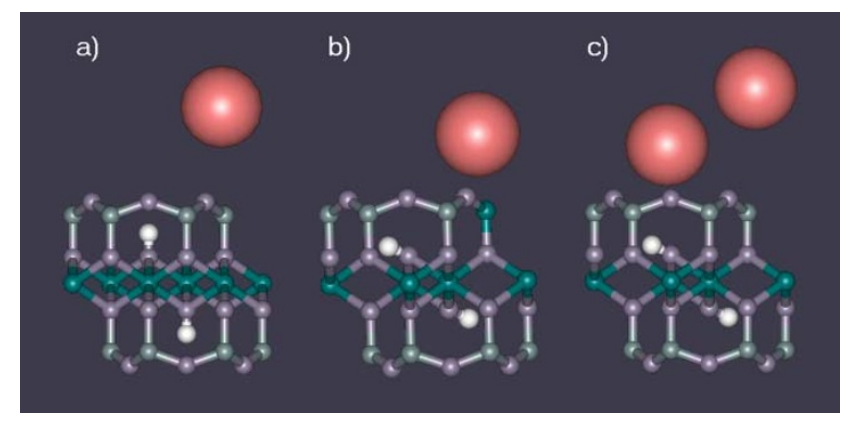

Fig. 4 Representations of preferred $\mathrm{Cs}^{+}$positions above six-oxygen cavities for (a) hectorite, (b) beidellite, and (c) montmorillonite. Interlayer species are represented as in Fig. 1. 
Although $\mathrm{Li}^{+}$ions within montmorillonite also displayed greater variation in position than their counterparts in hectorite and beidellite, they did not enter surface cavities, because these ions existed either as inner sphere surface complexes near oxygen triads, tightly bound to substitution sites, or as fully hydrated outer sphere complexes too large to enter a cavity. ${ }^{12}$ The difference in solvation of $\mathrm{Cs}^{+}$and $\mathrm{Li}^{+}$, a result of their contrasting ionic radii, thus modifies the electrostatic effect of the different smectite minerals.

It is possible that the ability to inhabit sites near oxygen triads as well as near or within six-oxygen cavities (Fig. 3), promotes the optimized geometry required for water sharing interactions to develop and perpetuate within the Csmontmorillonite interlayer over the timescale of our MD simulations. While $\mathrm{Cs}^{+}$within beidellite does not show this flexibility, the cations show another type of spatial variation, in that they are segregated into two layers along the $z$ axis (Fig. 3), one containing ions attracted to charge sites in the upper mineral layer, the other containing ions attracted to charge sites in the lower mineral layer. ${ }^{15}$ Although limited to positions close to oxygen triads, this vertical separation of cations may enhance the potential for sharing water molecules. In contrast, the charge of hectorite is located solely in the octahedral sheet, which compels $\mathrm{Cs}^{+}$ions to remain in stable, midplane positions near oxygen triads (Fig. 3), possibly inhibiting the formation of sharing configurations.

Extensive water sharing occurs in Cs-montmorillonite with $2 / 3$ water monolayer (Plate 4 ). In the lower right of the animation we see a complex consisting of four $\mathrm{Cs}^{+}$and five shared water molecules. This complex is stable over the 800 ps timespan of the simulation. The ions are located in close proximity to one tetrahedral and one octahedral charge site, and are near other tetrahedral and octahedral charge sites. In addition, we see the two $\mathrm{Cs}^{+}$not involved in the shared configuration roaming over the clay surface in a typical diffusional manner, unlike the hovering motions seen in the other systems. Plots of MSD vs. elapsed time for the two more mobile $\mathrm{Cs}^{+}$are nonlinear, ${ }^{15}$ which may indicate some form of entrapment. These $\mathrm{Cs}^{+}$can be seen to engage in ion exchange, as first one and then the other is attracted to an octahedral charge site submerged within the clay structure, located in the upper left corner of the simulation cell (Plate 4).

$\mathrm{Cs}^{+}$are not the only interlayer species attracted to specific

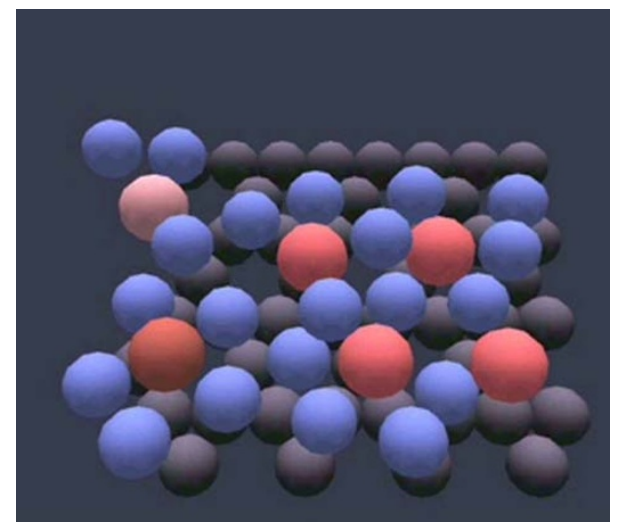

Plate 4 A 50 ps portion of the Cs-montmorillonite, 2/3 water monolayer MD simulation animation (750-800 ps). Interlayer species are represented as described in Plate 1 . The four $\mathrm{Cs}^{+}$toward the right of the animation are bound together by extended water sharing arrangements into a structure that is stable over the timespan of the simulation. The two $\mathrm{Cs}^{+}$toward the left of the simulation cell (red and brown) display continuous diffusional motion and participate in an exchange reaction, with first one and then the other taking up a position near an octahedral charge site in the clay layer. During the time that each cation is associated with this exchange site, a pale color is assigned to the relevant sphere. Click image or here to access the animation. sites within the clay mineral surface. Water molecules in Cs-hectorite with 1/3 water monolayer (Plate 1) frequently dip into the six-oxygen cavities, but rarely remain for more than a hundred picoseconds. The negative dipole of this interlayer species is attracted to the structural hydroxyl group of hectorite which rests at the bottom of each cavity (Fig. 4), creating a population of water molecules with their dipole moments pointing away from the mineral surface. Such a water orientation was found in MC simulations of Cshectorite. ${ }^{15}$ However, because the charges are small the interaction is not very strong. As a result, water molecules visit the cavities for tens of picoseconds or less. Simulations of Li-hectorite with low water content featured water molecules which inhabited cavities for the entire length of the 200 ps MD calculation, likely due to entrapment caused by the substantially smaller layer spacing of the mineral. ${ }^{12}$

Surface cavities receive more attention in Cs-beidellite (Plate 3) because the negative charge sites just below the mineral surface can attract the positive dipole of a water molecule just as they attract $\mathrm{Cs}^{+}$(Fig. 5). Water molecules so attracted arrange themselves with a hydrogen ion directed toward the clay surface, as seen in distributions of water dipolar orientation from MC calculations. ${ }^{15}$ The orientation of hydroxyl groups at a low angle relative to the plane of the clay surface ${ }^{42}$ allows some water molecules in the Csbeidellite simulation to remain in the surface cavities for hundreds of picoseconds. Simulations of Li-beidellite with a similarly low water content did not contain water molecules which sample the six-oxygen cavities. ${ }^{12}$ The Li-beidellite system had a larger layer spacing than that of Li-hectorite, which allowed all water molecules to coordinate around the strongly hydrating $\mathrm{Li}^{+}$ion. ${ }^{12}$

In Cs-montmorillonite with $1 / 3$ water monolayer, we see relatively little water entry into surface cavities (Plate 2). Because montmorillonite contains $1 / 3$ as much tetrahedral charge as beidellite, and its hydroxyl groups are oriented at a low angle with respect to the clay surface, ${ }^{42}$ the surface does not strongly attract either the positive or the negative dipoles of water molecules. The observation that $\mathrm{Cs}^{+}$within montmorillonite can enter six-oxygen cavities, unlike $\mathrm{Cs}^{+}$ within hectorite and beidellite, requires that cations outcompete water molecules for these locations. However, our MC results ${ }^{15}$ indicate that a portion of the water molecules within montmorillonite have a similar orientation to the fraction of water molecules in beidellite that are attracted to the mineral surface. Like Li-beidellite, Li-montmorillonite MD calculations did not show water molecules within six-oxygen cavities. ${ }^{12}$ This system also featured a larger layer spacing relative to

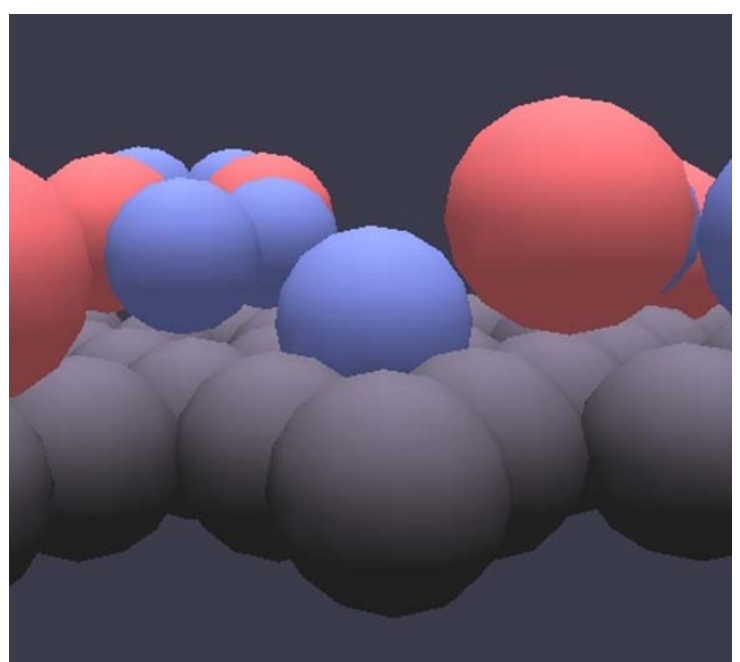

Fig. 5 Side view of a water molecule nestling into a six-oxygen cavity in the Cs-beidellite, 1/3 water monolayer system, as seen in Plate 3. 
Li-hectorite, allowing for more complete solvation of the strongly hydrating ions.

The amount of interlayer water modifies the extent of interaction of water molecules with the mineral surface. Animations of both Cs-montmorillonite and hectorite with 2/3 water monolayer (Plates 4 and 5) show a crowded interlayer featuring more extensive and lengthier sampling of the surface cavities by water molecules. As noted above, water movement in these systems is faster and more diffusional, and ions exhibit more rapid jump diffusion. Why do the $2 / 3$ water monolayer systems, though more tightly packed than the $1 / 3$ water monolayer systems, exhibit more movement among their interlayer species? Competition between ions and water molecules for surface sites may be the answer. An example of such competitive action takes place in the animation of the Cs-hectorite system (Plate 5). $\mathrm{A} \mathrm{Cs}^{+}$displaces a water molecule from a surface cavity near which it has hovered for tens of picoseconds. After only a few picoseconds, this cation is displaced in turn by another water molecule, as might be predicted given cation preference for locations over oxygen triads, rather than six-oxygen cavities, within hectorite. Similar and more subtle interactions across the clay surface would lead to greater movement of both water and $\mathrm{Cs}^{+}$. The electrostatic attraction between $\mathrm{Cs}^{+}$and the polar water molecules, responsible for the organization of the partial hydration shell, further encourages these competitive interactions by maintaining the two species in close proximity to each other as well as to the surface sites to which they are attracted.

\section{Conclusions}

Animations of 800 ps MD simulations have provided new information concerning the structure and dynamics of the hydrated Cs-smectite interlayer region. A comparison of the five animations presented here reveals the behavior of interlayer species as modified by water content and charge site location. Cs-smectite systems feature ions as inner sphere complexes, hovering near charge sites and occasionally using jump diffusional movements to relocate. Higher water contents encourage more rapid movement in the interlayer for both $\mathrm{Cs}^{+}$and the water molecules which solvate them. Different mineral charge patterns and structural hydroxyl group orientations create interlayer dynamics with different propensities for water molecule sharing between two $\mathrm{Cs}^{+}$. A low orientation angle for structural hydroxyl groups relative to the mineral surface, ${ }^{42}$ combined with a reduced amount of tetrahedral charge, as in montmorillonite, permits interlayer

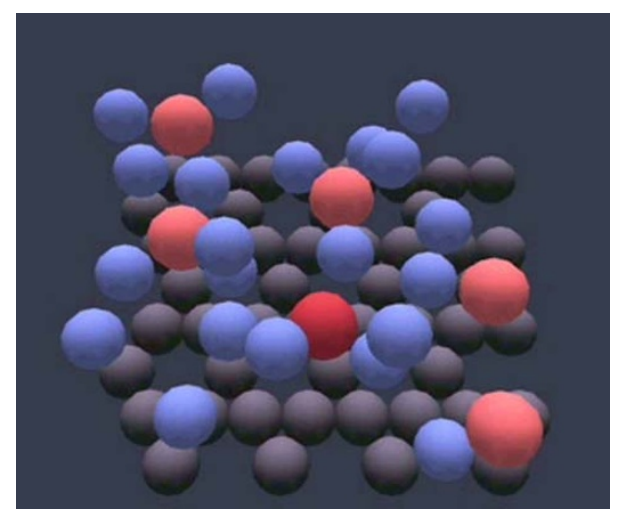

Plate 5 A 20 ps portion of the Cs-hectorite, 2/3 water monolayer MD simulation animation (625-645 ps). Interlayer species are represented as described in Plate 1. The highlighted $\mathrm{Cs}^{+}$(red) can be seen to compete with surrounding water molecules for favorable positions over six-oxygen cavities towards the end of this animation, when it moves forward to occupy a surface site closer to the viewer. Click image or here to access the animation. cations to occupy positions above and within six-oxygen cavities frequently. This may allow ions a greater flexibility in placement, and therefore increased ability to enter into water-sharing arrangements. Tetrahedrally charged clays like beidellite provide a different form of flexibility in ion position, due to the separation of ions into two layers along the $z$ axis. This segregation may encourage formation of water sharing arrangements as well. The combination of the octahedral charge and the perpendicular orientation of hydroxyl groups relative to the hectorite surface results in $\mathrm{Cs}^{+}$being held in stable, midplane locations above oxygen triads on the clay surface, hindering cations from entering into water sharing arrangements. The water sharing phenomenon predicted by our animations reaches an extreme state in the Cs-montmorillonite, 2/3 water monolayer hydrate, in which four $\mathrm{Cs}^{+}$are held together throughout the 800 ps simulation period in an extended complex of ions and shared water molecules. Our animations also document the attraction between water molecules and the clay mineral surface, which increases with higher water content. Animation reveals competition between water molecules and $\mathrm{Cs}^{+}$for sites on the mineral surface. Thus, animation of MD data has provided substantial information not available from traditional simulation methods.

\section{Acknowledgements}

Our research was supported in part by the Director, Office of Energy Research, Office of Basic Energy Sciences, Geosciences Division, of the U. S. Department of Energy under Contract No. DE-AC03-76SF00098. The National Energy Research Scientific Computing Center provided generous allocations of CPU time on its Cray J90 and T3E supercomputers. The first author expresses gratitude to the Clay Minerals Society for a grant supporting the purchase of a personal computer for use with her research, to Wes Bethel of the LBNL/NERSC Visualization Group for creating the customized RM Scene Graph program and for valuable discussions on visualization, and to Cristina Siegerist of the same group for help with the MediaConvert tool. Thanks also to the two anonymous reviewers who made valuable suggestions for the manuscript, and to reviewers John Apps and Satish Myneni of LBNL.

\section{References}

1 D. W. Evans, J. J. Alberts and R. A. Clark III, Reversible ionexchange fixation of cesium-137 leading to mobilization from reservoir sediments, Geochim. Cosmochim. Acta, 1983, 47, 1041.

2 W. J. Cho, D. W. Oscarson, M. N. Gray and S. C. H. Cheung, Influence of diffusant concentration on diffusion coefficients in clay, Radiochim. Acta, 1993, 60, 159.

3 D. E. Smith, Molecular computer simulations of the swelling properties and interlayer structure of cesium montmorillonite, Langmuir, 1998, 14, 5959.

4 D. J. Jensen and C. J. Radke, Caesium and strontium diffusion through sodium montmorillonite at elevated temperature, J. Soil Sci. $1988, \mathbf{3 9}, 53$.

5 D. W. Oscarson, H. B. Hume and F. King, Sorption of cesium on compacted bentonite, Clays Clay Miner., 1994, 42, 731.

6 Y. Onodera, T. Iwasaki, T. Ebina, H. Hayashi, K. Torii, A. Chatterjee and H. Mimura, Effect of layer charge on fixation of cesium ions in smectites, J. Contam. Hydrol., 1998, 35, 131.

7 T. Iwasaki and Y. Onodera, Sorption behaviour of caesium ions in smectites, in Proc. 10th Int. Clay Conf., Adelaide, Australia, 1993, ed. G. J. Churchman, R. W. Fitzpatrick and R. A. Eggleton, CSIRO Publishing, East Melbourne, Australia, 1995, p. 67.

8 G. Sposito, The Surface Chemistry of Soils, Oxford University Press, New York, 1984

9 D. M. C. MacEwan and M. J. Wilson, Interlayer and intercalation complexes of clay minerals, in Crystal Structures of Clay Minerals and their X-Ray Identification, ed. G. W. Brindley and G. Brown, Mineralogical Society, London, 1980, p. 197. 
10 F.-R. C. Chang, N. T. Skipper and G. Sposito, Computer simulation of interlayer molecular structure in sodium montmorillonite hydrates, Langmuir, 1995, 11, 2734.

11 F.-R. C. Chang, N. T. Skipper, K. Refson, J. A. Greathouse and G. Sposito, Interlayer molecular structure and dynamics in $\mathrm{Li}-, \mathrm{Na}$-, and K-montmorillonite-water systems, in Mineral-Water Interfacial Reactions: Kinetics and mechanisms, ed. D. L. Sparks and T. Grundl, American Chemical Society, Washington, DC, 1999, p. 88 .

12 J. Greathouse and G. Sposito, Monte Carlo and molecular dynamics studies of interlayer structure in $\mathrm{Li}\left(\mathrm{H}_{2} \mathrm{O}\right)_{3}$-smectites, J. Phys. Chem. B, 1998, 102, 2406.

13 N. T. Skipper, G. Sposito and F.-R. C. Chang, Monte Carlo simulation of interlayer molecular structure in swelling clay minerals. 2. Monolayer hydrates, Clays Clay Miner., 1995, 43, 294.

14 G. Sposito, N. T. Skipper, R. Sutton, S.-H. Park, A. K. Soper and J. A. Greathouse, Surface geochemistry of the clay minerals, Proc. Natl. Acad Sci. USA, 1999, 96, 3358.

15 R. Sutton and G. Sposito, Molecular simulation of interlayer structure and dynamics of $12.4 \AA$ Cs-smectite hydrates, J. Colloid Interface Sci., 2001, 237, 174

16 R. W. Mooney, A. G. Keenan and L. A. Wood, Adsorption of water vapor by montmorillonite. II. Effect of exchangeable ions and lattice swelling as measured by X-ray diffraction, J. Am. Chem. Soc., 1952, 74, 1371.

17 R. Calvet, Water adsorption on clays: Study of the hydration of montmorillonite, Bull. Soc. Chim. Fr., 1972, 8, 3097.

18 R. Prost, Interactions between adsorbed water molecules and the structure of clay minerals: Hydration mechanism of smectites, in Proc. 5th Int. Clay Conf., Mexico City, 1975, chief ed. S. W. Bailey, Applied Publishing, Wilmette, IL, 1976, p. 353.

19 I. Bérend, J. M. Cases, M. Francois, J. P. Uriot, L. Michot, A. Masion and F. Thomas, Mechanism of adsorption and desorption of water vapor by homoionic montmorillonites. 2. The $\mathrm{Li}^{+}, \mathrm{Na}^{+}, \mathrm{K}^{+}, \mathrm{Rb}^{+}$and $\mathrm{Cs}^{+}$-exchanged forms, Clays Clay Miner., 1995, 43, 324

20 C. T. Chiou and D. W. Rutherford, Effects of exchanged cation and layer charge on the sorption of water and EGME vapors on montmorillonite clays, Clays Clay Miner., 1997, 45, 867.

21 D. W. Rutherford, C. T. Chiou and D. D. Eberl, Effects of exchanged cation on the microporosity of montmorillonite, Clays Clay Miner., 1997, 45, 534.

22 R. Prost, Study of the hydration of clays: Water-mineral interactions and mechanism of water retention. II. Study of a smectite (hectorite), Ann. Agron., 1975, 26, 463.

23 C. Ware and G. Franck, Evaluating stereo and motion cues for visualizing information nets in three dimensions, Assoc. Comput. Mach. Trans. Graphics, 1996, 15, 121.

24 M. P. Allen and D. J. Tildesley, Computer Simulation of Liquids, Oxford University Press, Oxford, 1987
25 O. Matsuoka, E. Clementi and M. Yoshimine, CI study of the water dimer potential surface, J. Chem. Phys., 1976, 64, 1351.

26 D. E. Smith and L. X. Dang, Computer simulations of cesium water clusters - Do ion water clusters form gas-phase clathrates? J. Chem. Phys., 1994, 101, 7873.

27 N. T. Skipper, MONTE User's Manual, Department of Physics and Astronomy, University College London, UK, 1996.

28 K. Refson, MOLDY: A portable molecular dynamics simulation program for serial and parallel computers, Comput. Phys. Commun., 2000, 126, 310 .

29 The Visualization Group at Lawrence Berkeley National Laboratory and the National Energy Research Scientific Computing Center, http://vis.lbl.gov/.

30 OpenRM Scene Graph, http://openrm.sourceforge.net/.

31 G. Sposito, S.-H. Park and R. Sutton, Monte Carlo simulation of the total radial distribution function for interlayer water in sodium and potassium montmorillonites, Clays Clay Miner., 1999, 47, 192.

32 H. Ohtaki and T. Radnai, Structure and dynamics of hydrated ions, Chem. Rev., 1993, 93, 1157.

33 G. Sposito, Single-particle motions in liquid water. II. The hydrodynamic model, J. Chem. Phys., 1981, 74, 6943.

34 P. H. Nye, Diffusion of ions and uncharged solutes in soils and soil clays, Adv. Agron., 1979, 31, 225.

35 B. C. Bostick, M. A. Vairavamurthy, K. G. Karthikeyan and J. Chorover, Cesium adsorption on clay minerals: An EXAFS spectroscopic investigation, Environ. Sci. Technol., 2002, 36, 2670.

36 S. J. Anderson and G. Sposito, Cesium-adsorption method for measuring accessible structural surface charge, Soil Sci. Soc. Am. J., 1991, 55, 1569.

37 B. K. Schroth and G. Sposito, Surface charge properties of kaolinite, Clays Clay Miner., 1997, 45, 85.

38 C. A. Weiss, R. J. Kirkpatrick and S. P. Altaner, The structural environments of cations adsorbed onto clays: ${ }^{133} \mathrm{Cs}$ variabletemperature MAS NMR spectroscopic study of hectorite, Geochim. Cosmochim. Acta, 1990, 54, 1655.

39 C. A. Weiss, R. J. Kirkpatrick and S. P. Altaner, Variations in interlayer cation sites of clay minerals as studied by ${ }^{133} \mathrm{Cs}$ MAS nuclear magnetic resonance spectroscopy, Am. Mineral., 1990, 75, 970.

40 Y. Kim, R. J. Kirkpatrick and R. T. Cygan, ${ }^{133}$ Cs NMR study of Cs reaction with clay minerals, in Environmental Issues and Waste Management Techniques in the Ceramic and Nuclear Industry, ed. V. Jain and R. Palmer, American Ceramic Society, Westerville, OH, 1995, p. 629.

41 Y. Kim, R. T. Cygan and R. J. Kirkpatrick, ${ }^{133}$ Cs NMR and XPS investigation of cesium adsorbed on clay minerals and related phases, Geochim. Cosmochim. Acta, 1996, 60, 1041.

42 R. F. Giese, Jr., Hydroxyl orientations in $2: 1$ phyllosilicates, Clays Clay Miner., 1979, 27, 213 